\title{
Combining Ability Effects for De-husked Cob Yield in Baby Corn (Zea mays L.)
}

\author{
Sandeep Kumar Bangarwa* and R. B. Dubey
}

Department of Genetics and Plant Breeding, Rajasthan College of Agriculture, Maharana Pratap University of Agriculture and Technology, Udaipur-313001 (Raj.), India

*Corresponding author

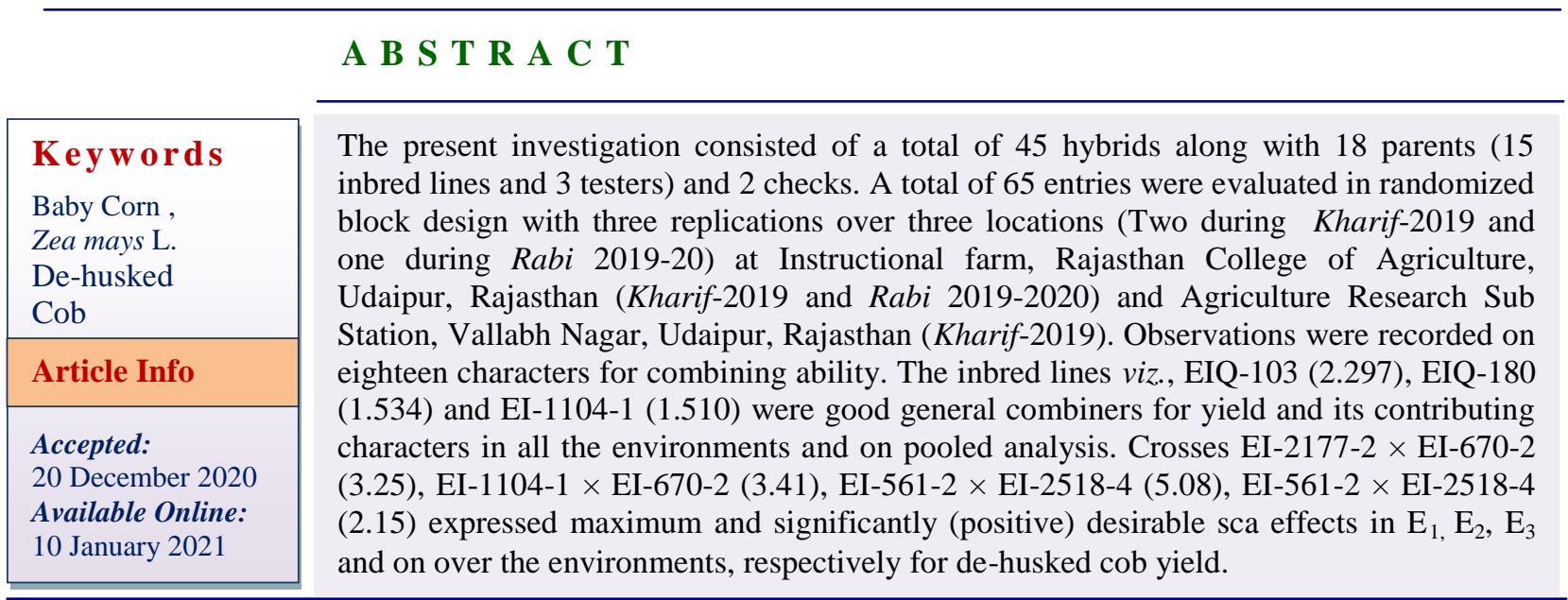

\section{Introduction}

Maize (Zea mays L.) $2 \mathrm{n}=20$, is the third most important cereal crop after rice and wheat in the world. By origin, maize is native to South America. Baby corn is a delicious, decorative and nutritious vegetable, without cholesterol. It is a low caloric vegetable which is rich in fibre content. One baby corn can be compared with an 'egg' in terms of minerals. Nutritive values of baby corn (per $100 \mathrm{~g}$ of edible portion) is Moisture $89.10 \%$, Carbohydrates $8.20 \mathrm{~g}$, Protein $1.90 \mathrm{~g}$, Calcium $28.00 \mathrm{mg}$, Phosphorus $86.00 \mathrm{mg}$, Iron $0.10 \mathrm{mg}$ (Jat et al., 2019).
Worldwide, Thailand is the leading producer and exporter of baby corn. India is emerging as the potential producer of baby corn due to high demand with less cost of production. In India, baby corn is being cultivated in Meghalaya, Western Uttar Pradesh, Haryana, Maharashtra, Karnataka and Andhra Pradesh. Young cob corn has been used by the Chinese as vegetable for generations, and this practice has spread to other Asian countries. It has nutritive value similar to that of non- legume vegetables such as cauliflower, cabbage, tomato, cucumber. This vegetable has versatile use after cooking and for processing as a canned product. 
India is emerging as one of the potential baby corn producing country due to low cost of production and high demand within the country. There is a great potential to earn foreign exchange through export of fresh/canned baby corn and its processed products. Another important point is that baby corn is a safe edible vegetable as it is almost free from residual effects of pesticides as the young cob on plant is wrapped with husk and well protected from insect attack and pathogens.

Baby corn has a short growing period (60-75 days), so that a farmer can grow four or more crops per year in a piece of land under tropical climatic conditions. It has a wide range of adaptation and does not need intensive practices for cultivation. The benefit of reaping with baby corn is that even after its harvest, the total herbage of maize plant can be utilized as green fodder. It generates more nutrition per unit area with the shortest crop duration and has the potential of being an excellent cash crop.

\section{Materials and Methods}

The present investigation consisted of a total of 45 hybrids along with 18 parents (15 inbred lines and 3 testers) and 2 checks. A total of 65 entries were evaluated in randomized block design with three replications over three locations (Two during Kharif-2019 and one during Rabi 2019-20) at Instructional farm, Rajasthan College of Agriculture, Udaipur, Rajasthan (Kharif-2019 and Rabi 2019-2020) and Agriculture Research Sub Station, Vallabh Nagar, Udaipur, Rajasthan (Kharif-2019). Observations were recorded on eighteen characters for combining ability. The experimental material comprised of 15 inbred lines viz.,EI-2311-4, EI-2449-2, EI-2403, EIQ-103, EIQ-104, EI-1104-1, EI-561-2, EI2173, EI-2177-2, EI-2509, EI-2518-2, EI-11-
3, EIQ-180, EIQ-225, EIQ-235 and 3 testers EI-670-2, EI-2518-4, EI-2156 their $45 \mathrm{~F}_{1 \mathrm{~s}}$ and two checks viz., HM-4 and VL Baby Corn-2. These $45 \mathrm{~F}_{1 \mathrm{~s}}$ were obtained by crossing 15 inbred lines and 3 testers in line $\times$ tester mating design. The combining ability effects for line $\mathrm{x}$ tester mating design was performed as per method suggested by Kemthorne (1957) for individual environments as well as over the environments.

\section{Combining ability effects for individual environment}

$$
\mu=\frac{\sum_{i=1}^{t} \sum_{j=1}^{l} \sum_{k=1}^{r} X_{i j k}}{l t r}
$$

$G C A$ line $=\frac{\sum_{i=1}^{t} \sum_{k=1}^{r} X_{i j k}}{t r}-\mu$

$G C A$ tester $=\frac{\sum_{i=1}^{l} \sum_{k=1}^{r} X_{i j k}}{l r}-\mu$

$S C A=\frac{\sum_{k=1}^{r} X_{i j k}}{r}-\frac{\sum_{j=1}^{t} \sum_{k=1}^{r} X_{i j k}}{t r}-\frac{\sum_{j=1}^{l} \sum_{k=1}^{r} X_{i j k}}{l r}+\mu$

Standard error of combining ability effects:

\begin{tabular}{|c|c|c|}
\hline S.E. (GCA line) & $=$ & $(\mathrm{MSE} / \mathrm{rt})^{1 / 2}$ \\
\hline S.E. (GCA tester) & $=$ & $(\mathrm{MSE} / \mathrm{rl})^{1 / 2}$ \\
\hline S.E. (SCA) & $=$ & $(\mathrm{MSE} / \mathrm{r})^{1 / 2}$ \\
\hline $\begin{array}{l}\text { S.E. }\left(\mathbf{G C A}_{\mathbf{i}}-\mathbf{G C A}_{\mathbf{i}}\right) \\
\text { line }\end{array}$ & $=$ & $(2 \times \mathrm{MSE} / \mathrm{rt})^{1 / 2}$ \\
\hline $\begin{array}{l}\text { S.E. }\left(\mathbf{G C A}_{\mathbf{i}}-\mathbf{G C A}_{\mathbf{i}}\right) \\
\text { tester }\end{array}$ & $=$ & $(2 \times \mathrm{MSE} / \mathrm{rl})^{1 / 2}$ \\
\hline S.E. $\left(\mathbf{S C A}_{i j}-\mathbf{S C A}_{\mathbf{k l}}\right)$ & $=$ & $(2 \times M S E / r)^{1 / 2}$ \\
\hline
\end{tabular}




\begin{tabular}{|c|c|c|}
\hline Where, & & \\
\hline $\mathbf{X}_{\mathrm{ijk}}$ & $=$ & $\begin{array}{l}\text { Value of hybrid between } \mathrm{i}^{\text {th }} \\
\text { and } \mathrm{j}^{\text {th }} \text { parent in } \mathrm{k}^{\text {th }} \\
\text { replication }\end{array}$ \\
\hline $\mathbf{t}$ & $=$ & Number of testers \\
\hline 1 & $=$ & Number of lines \\
\hline $\mathbf{r}$ & $=$ & Number of replications \\
\hline MSE & $=$ & Error mean square i.e. $\mathrm{M}_{14}$ \\
\hline
\end{tabular}

Over the environments general combining ability effects of parents and specific combining ability effects of hybrids were calculated for all the characters same manner as for individual environments except the number of environments was an additional divisor.

$\mu=\frac{\sum_{m=1}^{s} \sum_{i=1}^{r} \sum_{i=1}^{t} \sum_{k=1}^{r} X_{j l o n}}{s t r}$

GCA line $=\frac{\sum_{m=1}^{s} \sum_{i=1}^{t} \sum_{k=1}^{r} X_{i j k m}}{s t r}-\mu$

$G C A$ tester $=\frac{\sum_{m=1}^{s} \sum_{i=1}^{l} \sum_{k=1}^{r} X_{i j k m}}{s l r}-\mu$

\begin{tabular}{|l|l|l|}
\hline S.E. $(\mathbf{G C A}$ line) & $=(\mathrm{MSE} / \mathrm{rts})^{\mathbf{1 / 2}}$ \\
\hline S.E. $(\mathbf{G C A}$ tester$)$ & $=(\mathrm{MSE} / \mathrm{rls})^{1 / 2}$ \\
\hline S.E. $(\mathbf{S C A})$ & $=(\mathrm{MSE} / \mathrm{rs})^{1 / 2}$ \\
\hline S.E. $\left(\mathbf{G C A}_{\mathbf{i}}-\mathbf{G C A}_{\mathbf{j}}\right)$ line & $=(2 \mathrm{xSE} / \mathrm{rts})^{1 / 2}$ \\
\hline S.E. $\left(\mathbf{G C A}_{\mathbf{i}}-\mathbf{G C A}_{\mathbf{j}}\right)$ tester & $=(2 \mathrm{xSE} / \mathrm{rls})^{1 / 2}$ \\
\hline S.E. $\left(\mathbf{S C A}_{\mathbf{i j}}-\mathbf{S C A}_{\mathbf{k l}}\right)$ & $=(2 \mathrm{xSE} / \mathrm{rs})^{1 / 2}$ \\
\hline S.E. $\left(\mathbf{G C A}_{\mathbf{i j}}-\mathbf{G C A}_{\mathbf{i}}\right)$ line & $=[(1+\mathrm{s}) \mathrm{MSE} / \mathrm{rts}]^{1 / 2}$ \\
\hline S.E. $\left(\mathbf{G C A}_{\mathbf{i j}}-\mathbf{G C A}_{\mathbf{i}}\right)$ tester & $=[(1+\mathrm{s}) \mathrm{MSE} / \mathrm{rls}]^{1 / 2}$ \\
\hline S.E. $\left(\mathbf{S C A}_{\mathbf{i j k}}-\mathbf{S C A}_{\mathbf{i j}}\right)$ & $=[(1+\mathrm{s}) \mathrm{MSE} / \mathrm{rs}]^{1 / 2}$ \\
\hline
\end{tabular}

\begin{tabular}{|l|l|l|}
\hline Where, & & \\
\hline $\mathbf{X}_{\mathbf{i j k m}}$ & $=$ & $\begin{array}{l}\text { Value of hybrid between } \mathrm{i}^{\text {th }} \text { and } \mathrm{j}^{\text {th }} \text { parent } \\
\text { in } \mathrm{k}^{\text {th }} \text { replication and } \mathrm{m}^{\text {th }} \text { environment. }\end{array}$ \\
\hline $\mathbf{t}$ & $=$ & Number of testers \\
\hline $\mathbf{l}$ & $=$ & Number of lines \\
\hline $\mathbf{r}$ & $=$ & Number of replications \\
\hline $\mathbf{S}$ & $=$ & Number of environments \\
\hline MSE & $=$ & Error mean square i.e. $\mathrm{M}_{27}$ \\
\hline
\end{tabular}

\section{Results and Discussion}

Two lines (EI-1104-1 and EIQ-180) in $\mathrm{E}_{1}$, Five lines (EI-2403, EIQ-103, EIQ-104, EIQ-180 and EIQ-235) in $\mathrm{E}_{2}$, four lines (EIQ-103, EI1104-1, EI-2173 and EI-11-3) in $\mathrm{E}_{3}$ and three lines (EIQ-103, EI-1104-1 and EIQ-180) on over the environments, exhibited significantly positive gca effects for de-husked cob yield per plant. In $E_{1}$, female line EIQ-180 (1.88) showed significantly maximum positive desirable gca effects followed by EI-1104-1 (1.64). In $\mathrm{E}_{2}$, the female line EIQ-104 (3.06) expressed maximum positive GCA effects folloried $X$ by EIQ-235 (2.59) and EIQ-103 द.7.7. In ${ }_{k=1}$, the female line EIQ-103 (3.19) exhipited maxinkum positive GCA effects folfowed by EI-1104-1 (2.35) and EI-2173 (1.88). On pooled basis, the female line EIQ103 (2.29) indicated maximum positive gca effects followed by EIQ-180 (1.53) and EI1104-1 (1.51). None of the male parent in any environment was found with positive significant gca effects for de-husked cob yield per plant.

The standard error of effects was worked out as follows: 
Table.1 Analysis of variance for combining ability on over the environments

\begin{tabular}{|c|c|c|c|c|c|c|c|c|c|c|c|c|c|c|c|c|c|c|c|}
\hline \multirow[b]{2}{*}{$\begin{array}{l}\text { Source of } \\
\text { variation }\end{array}$} & \multirow[b]{2}{*}{$\begin{array}{l}\text { d. } \\
\text { f. }\end{array}$} & \multicolumn{18}{|c|}{ Mean squares } \\
\hline & & 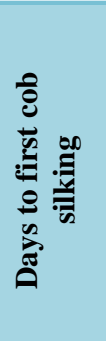 & 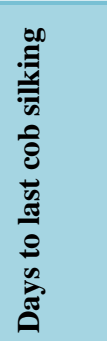 & 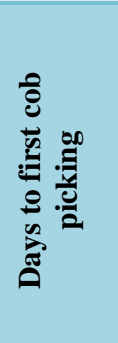 & 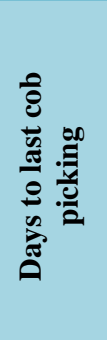 & 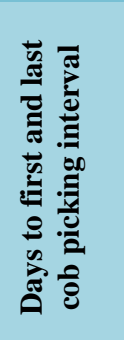 & 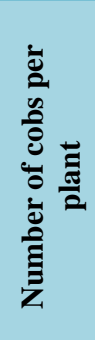 & 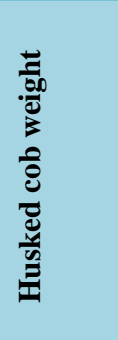 & 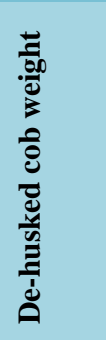 & 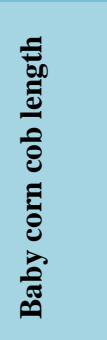 & 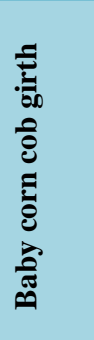 & 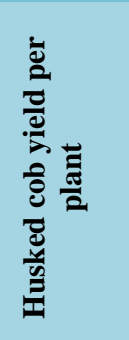 & 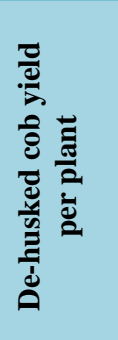 & 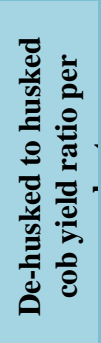 & 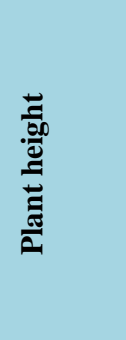 & 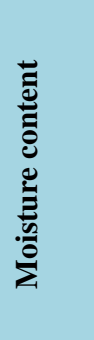 & 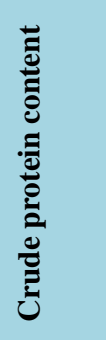 & की & 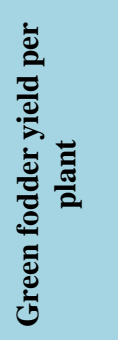 \\
\hline $\begin{array}{l}\text { Replications } \\
\text { (r) }\end{array}$ & 2 & 0.906 & 79.055 & $8.718^{*}$ & $\begin{array}{c}10.476 \\
* *\end{array}$ & 0.195 & 0.11 & 1.133 & 0.066 & 0.726 & 0.229 & 2.921 & 1.82 & 0 & 56.461 & 2.322 & 0.432 & 0.531 & $\begin{array}{c}3840.43 \\
6^{*}\end{array}$ \\
\hline $\begin{array}{l}\text { Environment } \\
\text { s (e) }\end{array}$ & 2 & $\begin{array}{c}12133 . \\
4 * *\end{array}$ & $\begin{array}{c}21508 . \\
6 * *\end{array}$ & $\begin{array}{c}13846.3 \\
4 * *\end{array}$ & $\begin{array}{c}27315 . \\
1 * *\end{array}$ & $\begin{array}{c}2357.66 \\
2 * *\end{array}$ & $\begin{array}{c}5.763 \\
* *\end{array}$ & $\begin{array}{c}2662.07 \\
1 * *\end{array}$ & $\begin{array}{c}101.02 \\
8 * *\end{array}$ & $\begin{array}{c}124.68 \\
4 * *\end{array}$ & $\begin{array}{c}63.32 \\
2 * *\end{array}$ & $\begin{array}{c}59280.7 \\
* *\end{array}$ & $\begin{array}{c}2137.05 \\
8 * *\end{array}$ & $\begin{array}{c}0.001 \\
* *\end{array}$ & $\begin{array}{c}18373.5 \\
1 * *\end{array}$ & $\begin{array}{c}95.95 \\
8 * *\end{array}$ & $\begin{array}{c}118.10 \\
4 * *\end{array}$ & 0.486 & $\begin{array}{c}1038739 \\
* *\end{array}$ \\
\hline Rep. $\times$ Env. & 4 & 9.939 & 23.75 & 4.088 & 1.83 & 1.143 & 0.01 & 4.796 & 0.292 & 0.07 & 0.179 & 9.187 & 3.043 & 0 & 43.654 & 1.167 & 0.167 & 0.413 & 754.27 \\
\hline Crosses & 44 & $\begin{array}{c}22.967 \\
* *\end{array}$ & 29.382 & $\begin{array}{c}14.521 * \\
*\end{array}$ & $\begin{array}{c}11.390 \\
* *\end{array}$ & $\begin{array}{c}14.859 * \\
*\end{array}$ & $\begin{array}{c}0.117 \\
* *\end{array}$ & $\begin{array}{c}71.290 * \\
*\end{array}$ & $\begin{array}{c}2.034 * \\
*\end{array}$ & $\begin{array}{c}1.948 * \\
*\end{array}$ & $\begin{array}{c}0.494 \\
* *\end{array}$ & $\begin{array}{c}766.358 \\
* *\end{array}$ & $\begin{array}{c}22.093 * \\
*\end{array}$ & 0 & $\begin{array}{c}736.222 \\
* *\end{array}$ & $\begin{array}{c}10.10 \\
2 * *\end{array}$ & $2.30 * *$ & $\begin{array}{c}4.051 \\
* *\end{array}$ & $\begin{array}{c}20336.9 \\
40 * *\end{array}$ \\
\hline line (I) & 14 & 30.722 & 33.009 & 22.379 & 15.634 & 20.134 & $\begin{array}{c}0.189 \\
*\end{array}$ & $\begin{array}{c}134.611 \\
*\end{array}$ & $\begin{array}{c}3.679 * \\
*\end{array}$ & $\begin{array}{c}3.465 * \\
*\end{array}$ & 0.686 & $\begin{array}{c}1373.18 \\
2 * *\end{array}$ & $\begin{array}{c}43.456^{*} \\
*\end{array}$ & 0 & $\begin{array}{c}1892.39 \\
0 * *\end{array}$ & $\begin{array}{c}16.11 \\
4 *\end{array}$ & $\begin{array}{c}5.530 * \\
*\end{array}$ & $\begin{array}{c}7.238 \\
*\end{array}$ & $\begin{array}{c}52330.4 \\
60 * *\end{array}$ \\
\hline tester $(t)$ & 2 & 12.458 & 1.819 & 2.539 & 26.859 & $38.866^{*}$ & 0.02 & 37.616 & 0.767 & $4.472 *$ & 0.889 & 561.505 & 10.739 & 0 & 41.873 & 1.852 & 0.638 & 0.473 & 10642.8 \\
\hline line $\times$ tester & 28 & $\begin{array}{c}19.840 \\
* *\end{array}$ & 29.541 & $\begin{array}{c}11.449 * \\
*\end{array}$ & $\begin{array}{c}8.163 * \\
*\end{array}$ & $\begin{array}{c}10.507 * \\
*\end{array}$ & $\begin{array}{c}0.087 \\
* *\end{array}$ & $\begin{array}{c}47.035^{*} \\
*\end{array}$ & $\begin{array}{c}1.303 * \\
*\end{array}$ & $\begin{array}{c}1.010^{*} \\
*\end{array}$ & $\begin{array}{c}0.370 \\
* *\end{array}$ & $\begin{array}{c}477.578 \\
* *\end{array}$ & $\begin{array}{c}12.222 * \\
*\end{array}$ & 0 & $\begin{array}{c}207.735 \\
* *\end{array}$ & $\begin{array}{c}7.685 \\
* *\end{array}$ & $\begin{array}{l}0.804 * \\
*\end{array}$ & $\begin{array}{c}2.712 \\
* *\end{array}$ & $\begin{array}{c}5032.62 \\
8 * *\end{array}$ \\
\hline $\begin{array}{l}\text { Environment } \\
\times \text { Crosses }\end{array}$ & 88 & $\begin{array}{c}17.98^{*} \\
*\end{array}$ & $\begin{array}{c}37.808 \\
*\end{array}$ & $\begin{array}{c}15.125^{*} \\
*\end{array}$ & $\begin{array}{c}10.666 \\
* *\end{array}$ & $\begin{array}{c}13.173 * \\
*\end{array}$ & $\begin{array}{c}0.137 \\
* *\end{array}$ & $\begin{array}{c}43.711 * \\
*\end{array}$ & $\begin{array}{c}1.330 * \\
*\end{array}$ & $\begin{array}{c}1.603 * \\
*\end{array}$ & $\begin{array}{c}0.519 \\
* *\end{array}$ & $\begin{array}{c}649.470 \\
* *\end{array}$ & $\begin{array}{c}21.175 * \\
*\end{array}$ & $\begin{array}{c}0.00 * \\
*\end{array}$ & $\begin{array}{c}271.695 \\
* *\end{array}$ & $\begin{array}{c}8.282 \\
* *\end{array}$ & $\begin{array}{c}3.673 * \\
*\end{array}$ & $\begin{array}{c}4.457 \\
* *\end{array}$ & $\begin{array}{c}20971.1 \\
70^{* *}\end{array}$ \\
\hline $\begin{array}{l}\text { Environment } \\
\times \text { Line } \\
\text { effects }\end{array}$ & 28 & 21.163 & $\begin{array}{c}57.070 \\
*\end{array}$ & $\begin{array}{c}27.167 * \\
*\end{array}$ & $\begin{array}{c}19.554 \\
* *\end{array}$ & $\begin{array}{c}22.126 * \\
*\end{array}$ & $\begin{array}{c}0.244 \\
* *\end{array}$ & $\begin{array}{c}74.234 * \\
*\end{array}$ & $\begin{array}{c}2.520^{*} \\
*\end{array}$ & $\begin{array}{c}2.550 * \\
*\end{array}$ & $\begin{array}{c}0.857 \\
* *\end{array}$ & $\begin{array}{c}941.536 \\
*\end{array}$ & $31.122 *$ & $\begin{array}{c}0.00 * \\
*\end{array}$ & $\begin{array}{c}629.133 \\
* *\end{array}$ & $\begin{array}{c}10.20 \\
5 * *\end{array}$ & $\begin{array}{c}9.498 * \\
*\end{array}$ & $\begin{array}{c}8.123 \\
* *\end{array}$ & $\begin{array}{c}49034.5 \\
8 * *\end{array}$ \\
\hline $\begin{array}{l}\text { Environment } \\
\times \text { tester } \\
\text { effects }\end{array}$ & 4 & 21.582 & 22.138 & 21.691 & 4.408 & 20.629 & 0.025 & 9.518 & 0.231 & 2.158 & 0.26 & 86.212 & 4.472 & 0 & 38.254 & 1.918 & 0.738 & 3.804 & $\begin{array}{c}3533.93 \\
6\end{array}$ \\
\hline $\begin{array}{l}\text { Environment } \\
\times \text { Line } \times \\
\text { tester effects }\end{array}$ & 56 & $\begin{array}{c}14.631 \\
* *\end{array}$ & 29.296 & $8.635^{* *}$ & $\begin{array}{c}6.669 * \\
*\end{array}$ & $8.164 * *$ & $\begin{array}{c}0.092 \\
* *\end{array}$ & $\begin{array}{c}30.891 * \\
*\end{array}$ & $\begin{array}{c}0.813 * \\
*\end{array}$ & $\begin{array}{c}1.089 * \\
*\end{array}$ & $\begin{array}{c}0.368 \\
* *\end{array}$ & $\begin{array}{c}543.669 \\
* *\end{array}$ & $\begin{array}{c}17.394 * \\
*\end{array}$ & $\begin{array}{c}0.000 \\
*\end{array}$ & $\begin{array}{c}109.651 \\
* *\end{array}$ & $\begin{array}{c}3.755 \\
* *\end{array}$ & $\begin{array}{c}0.971 * \\
*\end{array}$ & $\begin{array}{c}2.671 \\
* *\end{array}$ & $\begin{array}{c}8184.97 \\
6 * *\end{array}$ \\
\hline Error & $\begin{array}{c}26 \\
4\end{array}$ & 7.849 & 27.741 & 2.386 & 2.092 & 2.821 & 0.026 & 4.908 & 0.286 & 0.366 & 0.146 & 99.547 & 4.347 & 0 & 42.851 & 2.119 & 0.228 & 0.486 & 852.714 \\
\hline Total & $\begin{array}{c}40 \\
4\end{array}$ & 71.716 & $\begin{array}{c}136.66 \\
8\end{array}$ & 75.065 & $\begin{array}{c}140.22 \\
4\end{array}$ & 18.015 & 0.088 & 33.724 & 1.201 & 1.422 & 0.579 & 583.558 & 20.477 & 0 & 259.035 & 4.787 & 1.788 & 1.739 & $\begin{array}{c}12508.8 \\
6\end{array}$ \\
\hline
\end{tabular}


One cross (EI-2177-2 $\times$ EI-670-2) in $E_{1}$, six crosses (EI-2311-4 × EI-2518-4, EI-2403 $\times$ EI-2156, EI-1104-1 × EI-670-2, EI-2509 $\times$ EI-670-2, EI-2518-2 $\times$ EI-2518-4 and EI-11-3 $\times$ EI-670-2) in $\mathrm{E}_{2}$, five crosses (EI-2311-4 $\times$ EI-2156, EI-561-2 × EI-2518-4, EI-2173 × EI-670-2, EI-11-3 $\times$ EI-2156 and EIQ-235 $\times$ EI-670-2) in $\mathrm{E}_{3}$ and two crosses (EIQ-103 $\times$ EI-2156 and EI-561-2 $\times$ EI-2518-4) on pooled analysis, showed significantly positive sca effects for de-husked cob yield per plant. Maximum significantly positive sca effects were depicted by cross EI-2177-2 $\times$ EI-670-2 (3.25) in $\mathrm{E}_{1}$. In $\mathrm{E}_{2}$, cross EI-1104-1 $\times$ EI-6702 (3.41) expressed maximum significantly positive desirable sca effects for this trait followed by EI-11-3 × EI-670-2 (2.59) and EI-2509 $\times$ EI-670-2(2.49). In $\mathrm{E}_{3}$, cross EI561-2 × EI-2518-4 (5.08) showed maximum significant positive sca effects for this trait followed by EI-2311-4 × EI-2156 (3.38) and EI-2173 $\times$ EI-670-2(2.81). On pooled basis, crossEI-561-2 × EI-2518-4 (2.15) indicated maximum significantly positive desirable sca effects followed by EIQ-103 × EI-2156
(1.40). In the present study, combining ability indicated that mean sum of squares due to crosses were significant for all the traits in all the environments as well as over the environments (Table-1). Partitioning of these mean sum of squares into lines, testers and line $\mathrm{x}$ tester interaction revealed that the mean sum of squares due to line, tester and line $\mathrm{x}$ tester were found to be significant for most of the traits in all the environments as well as in data pooled over environments.

\section{References}

Jat, S.L., Parihar, C.M., Preeti, Radheshyam, Kumar, B., Chikkappa G.K. and Singh, A.K. 2019. Baby corn and sweet corn production technologies for Mizoram. National workshop on Scientific Maize Cultivation in North East India, 5th March 2019, Aizawl, Mizoram: 8-21.

Kempthorne, O. 1957. An introduction to genetic statistics. John Wiley and Sons, Inc., New York.

\section{How to cite this article:}

Sandeep Kumar Bangarwa and Dubey, R. B. 2021. Combining Ability Effects for De-husked Cob Yield in Baby Corn (Zea mays L.). Int.J.Curr.Microbiol.App.Sci. 10(01): 3545-3549. doi: https://doi.org/10.20546/ijcmas.2021.1001.418 